\title{
Experience in using anisotropic properties of composites in engineering the compressor impeller of a small-size gas turbine engine
}

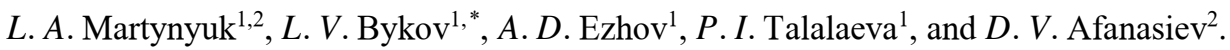 \\ ${ }^{1}$ Moscow Aviation Institute (National Research University), Moscow, Russia \\ ${ }^{2}$ Central Institute of Aviation Motors, Moscow, Russia
}

\begin{abstract}
The use of composite materials in modern aircraft and rocket engines is one of the most promising areas. Low density and high strength characteristics of composite materials are crucial when choosing a material for small-sized compressors. The main ability to bear the load of the composite material is provided by the reinforcing fibers of the filler. The greater the percentage of filler fibers has a particular orientation, the higher the strength and rigidity of the product in this direction. If the part is loaded with forces applied primarily in one or two directions, it makes sense to create a material with anisotropy of properties that will exactly match the applied loads. For example, the disk and blades of a centrifugal compressor operate under the action of centrifugal force and gas pressure. In this case, to manufacture a centrifugal compressor impeller from composite materials, it is only necessary to redistribute the fibers in the part space in such a way as to create an optimal anisotropy of properties. This article describes the procedure for selecting the optimal orientation of reinforcing fibers in the impeller of a centrifugal compressor of a small gas turbine engine.
\end{abstract}

\section{Introduction}

The growth of the scientific and technical potential of modern engine building is inseparably linked with the development of technologies for obtaining new structural materials, and methods for mathematical modeling of the properties of future products in order to reduce the cost of developing and improving real components and assemblies. The most promising and relatively new, at present, are composite materials that allow you to fully implement design ideas and improve the performance of small-sized gas turbine engines. The transition to composite materials in the engine industry can be compared to the revolution in aircraft construction in the transition from wood to metal. Scientific and practical interest in the problem of using composite materials for the manufacture of compressor impellers for aircraft engines is primarily due to the unique physical and mechanical properties of such materials. Thus, in the rotor Assembly of a small gas turbine engine, the use of a lightweight centrifugal compressor impeller made of a high-

\footnotetext{
* Corresponding author: bykovlv@mai.ru
} 
temperature polymer composite material will reduce their own weight, reduce the load on the bearings and, as a result, increase the engine life as a whole.

Special attention should be paid to calculating the thermal state of the impeller disk of a centrifugal compressor. Thus, when the compressor is running, a zone of significant overheating may occur on the back surface of the disk [1], which can lead to the destruction of the binder, as well as degradation of the strength properties of the material [2]. The article [3] focuses on the effect of heat load on the strength of the compressor impeller by comparing the results that take into account only the centrifugal load with the results that take into account both the centrifugal and thermal load at different degrees of air compression. The surface temperature of the compressor impeller is obtained by calculating the heat transfer elements across the interface between the solid surface of the wheel and the gas. The values of the temperature field at the interface obtained by the finite element method are taken as boundary conditions for conducting thermal analysis, the results of which are used in calculating the stress state of the structure. The centrifugal load is determined by the number of revolutions of the compressor impeller. The considered method does not take into account the thermal state of the back surface of the compressor disk, so it cannot fully provide data on the degradation of the strength properties of materials under the influence of heat load.

Analysis of previously known designs of centrifugal compressors leads to the conclusion that all designs can be divided into two classes: the first class of compressors made of polymer composite materials implies mechanical processing of a single composite billet, the second class implies the Assembly of the compressor from individual parts, such as blades, disks, power rings, inserts, etc. When designing and manufacturing first class compressors, it is not possible to create a fiber-laying structure that would be optimal in terms of strength. The impellers of the first class practically do not contain fibers directed radially (relative to the axis of rotation of the wheel) and perceive the main centrifugal load, which leads to their destruction at high speeds. The fact is that this technology is based on well-known metal processing technologies, which allows you to use standard equipment and a lot of accumulated experience in this field. The second class of compressors allows you to create an optimal structure for the location of fibers inside the part, individual for each type of element, in terms of strength and rigidity. All manufactured parts are thoroughly checked. Products that have not passed the control are rejected. This allows you to reduce costs in the case of manufacturing defects of individual elements. The main disadvantage of this method is an increase in the number of technological operations for monitoring and assembling the impeller of a centrifugal compressor. It requires the production of complex and expensive assembly equipment. However, the most advantageous from the point of view of creating complex geometric configurations, ensuring their strength and rigidity is the second class of impellers, which was chosen as the object of research in this work.

\section{Object of research}

The impeller of a centrifugal compressor of a small-sized gas turbine engine made of titanium alloy intended for installation on unmanned aerial vehicles. The initial parameters of the centrifugal compressor impeller are shown in the table. 1 and table. 2.

Table 1. Parameters of the centrifugal compressor impeller

\begin{tabular}{|c|c|c|c|c|c|}
\hline $\begin{array}{c}\eta \\
\mathrm{rpm}\end{array}$ & \multirow{\mathrm{G}}{*}{$\mathrm{kg} / \mathrm{s}$} & \multicolumn{2}{|c|}{ Inlet } & \multicolumn{2}{|c|}{ Outlet } \\
\cline { 3 - 6 } & $\mathrm{T}_{\text {in }}^{*}, \mathrm{~K}$ & $P_{\text {in }}^{*}$, bar & $\mathrm{T}_{\text {out }}^{*}, \mathrm{~K}$ & $P_{\text {out }}^{*}$, bar \\
\hline 52000 & 2,1 & 350 & 2 & 558 & 7 \\
\hline
\end{tabular}


Table 2. Main geometrical dimensions of the centrifugal compressor impeller

\begin{tabular}{|c|c|c|c|}
\hline \multicolumn{2}{|c|}{ inlet } & \multirow{2}{*}{$\mathrm{D}, \mathrm{mm}$} & \multirow{2}{*}{$\mathrm{b}, \mathrm{mm}$} \\
\cline { 1 - 2 } $\mathrm{d}_{\text {internal, }} \mathrm{mm}$ & $\mathrm{d}_{\text {outer }}, \mathrm{mm}$ & 176 & 68,1 \\
\hline 45 & 106,2 & 176 & 6 \\
\hline
\end{tabular}

The maximum operating time at maximum $\mathrm{rpm} \eta=52,000 \mathrm{rpm}$ is 3 hours based on the purpose of the engine. As part of the research, the task is to make a prototype of the impeller of a centrifugal compressor from a polymer composite material and equip the engine with it.

\subsection{Engine description}

The engine is intended for installation on unmanned aerial vehicles. It is a short-term turbojet engine. It has a two-stage (axial and centrifugal) compressor that directs the air flow through a radial diffuser and a guide device to the annular combustion chamber. Fuel is fed into the chamber through eight injectors. The single-stage axial turbine is directly connected to the compressor. The shaft is supported by four bearings. Lubrication is carried out by fuel. The engine is started by compressed air supplied from an external source. The fuel pump and generator are driven from the engine shaft. Engine operation is provided by an electronic control system.

\section{Determination of thermophysical properties of carbon fiber}

Determining reliable thermophysical properties of carbon fiber is a complex multi-factor task. On thermal properties of carbon are affected: thermophysical properties of a reinforcing material, the thermal properties of the binder, the stacking direction of the reinforcing material, extrusion technology products, volumetric binder content, porosity, foreign inclusions [4]. The method of mathematical modeling of the heat transfer process in a unit cell of a layered polymer composite material [5] does not take into account defects that occur during the production of the product (the presence of pores, foreign inclusions). The mixture rule [6] allows only approximate calculation of the properties of the future material. Therefore, in this study, preference is given to the experimental determination of the thermophysical properties of carbon fiber. For experimental determination of thermal conductivity, material work pieces were made with two types of reinforcement material laying: [0] n; [0;+45;0;-45]n (Fig. 1), based on ITECMA UMT-49s reinforcing fibers and $\mathrm{PN}-3 \mathrm{M}$ phthalonitrile binder. Then experimental samples were cut from the work pieces "tablets "in mutually perpendicular directions $-\mathrm{X}, \mathrm{Y}, \mathrm{Z}$, where $\mathrm{X}$ is the direction of laying the reinforcing material, $\mathrm{Y}$ is the transversal direction in the layer plane, and $\mathrm{Z}$ is the" third " direction (along the thickness of the sample). Stacking of type [0]n characterizes the properties of a single layer. It is a passport characteristic of a composite material and can be directly used in "layered" models of a material of a high level of complexity. At the first stage of strength design, you can use more simple models for describing a composite material, for example, the model of a solid orthotropic body, in which the properties of the material in the macro volume are averaged in three directions. 
Fig. 1. Material work pieces with two types of reinforcement material laying.

Taking into account the experience of designing prototype products, for the initial calculation of the centrifugal wheel, it is assumed that the layers in such a product are laid according to the scheme $[0 ;+45 ; 0 ;-45]$ n. Having made samples of the material with such laying, you can experimentally determine all the necessary characteristics. In the event that the initial strength project shows that the laying $[0 ;+45 ; 0 ;-45]$ if $\mathrm{n}$ is not optimal, then change the rotation angles of the layers and repeat the strength calculation. In this case, the thermal characteristics of the package should be obtained by calculation, using the thermal conductivity of the unidirectional layer [0] $\mathrm{n}$ and setting the optimal reinforcement angles. To do this, use the methods for calculating the above characteristics described in [5] and [6].

To study the thermal conductivity of the material, the laser flash method (the Parker method) was used, the scheme of which is shown in Fig.2. The study was conducted on the installation of the NETZSCH LFA 457 MicroFlash.

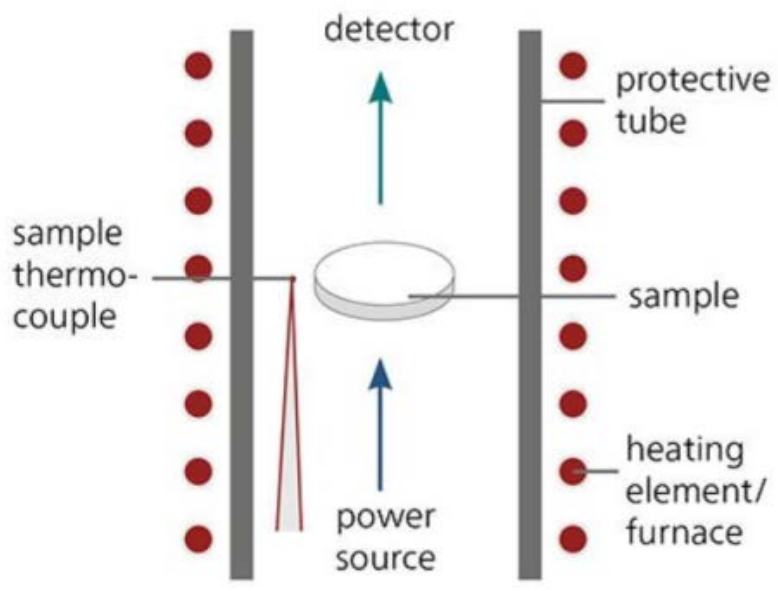

Flash Technique

Fig. 2. Essence of the laser flash method. 
As a result of tests, thermal conductivity was obtained in three directions for laying $[0 ;+45 ; 0 ;-45] n$ (Table 3 ) and for laying [0]n (Table 4)

Table 3. Thermal conductivity of carbon fiber with laying $[0 ;+45 ; 0 ;-45] \mathrm{n}$

\begin{tabular}{|c|c|c|c|}
\hline $\mathbf{T},{ }^{\mathbf{0}} \mathbf{C}$ & $\boldsymbol{\lambda}_{\mathbf{x}}, \mathbf{W} /\left(\mathbf{m}^{*} \mathbf{K}\right)$ & $\boldsymbol{\lambda}_{\mathbf{y}}, \mathbf{W} /(\mathbf{m} * \mathbf{K})$ & $\boldsymbol{\lambda}_{\mathbf{z}}, \mathbf{W} /(\mathbf{m} * \mathbf{K})$ \\
\hline 20 & 5,196 & 1,998 & 0,682 \\
\hline 200 & 8,267 & 3,033 & 0,97 \\
\hline
\end{tabular}

Table 4. Thermal conductivity of carbon fiber with laying [0]n

\begin{tabular}{|c|c|c|c|}
\hline $\mathbf{T},{ }^{\mathbf{0}} \mathbf{C}$ & $\boldsymbol{\lambda}_{\mathbf{x}}, \mathbf{W} /(\mathbf{m} * \mathbf{K})$ & $\boldsymbol{\lambda}_{\mathbf{y}}, \mathbf{W} /\left(\mathbf{m}^{*} \mathbf{K}\right)$ & $\boldsymbol{\lambda}_{\mathbf{z}}, \mathbf{W} /(\mathbf{m} * \mathbf{K})$ \\
\hline 20 & 5,291 & 0,929 & 0,578 \\
\hline 200 & 7,279 & 1,189 & 0,713 \\
\hline
\end{tabular}

\section{Technology for manufacturing the impeller of a centrifugal compressor of a small-sized gas turbine engine by the method of directional laying of reinforcing material}

As described earlier, the second class of compressor was selected, so the impeller of a centrifugal compressor is divided into 20 blade segments (Fig.3) and 5 power rings.

For the production of blade segments (Fig. 3), the technology of reinforcing filler stripe was chosen, which can be implemented using specialized embroidery machines equipped with CNC, allowing you to sew roving (straight) fibers of various types, including carbon, on a flat fabric substrate. Such two-coordinate machines allow you to lay the fiber on the table plane along a complex trajectory, simultaneously sewing it to the base with an auxiliary thread (Fig. 4).

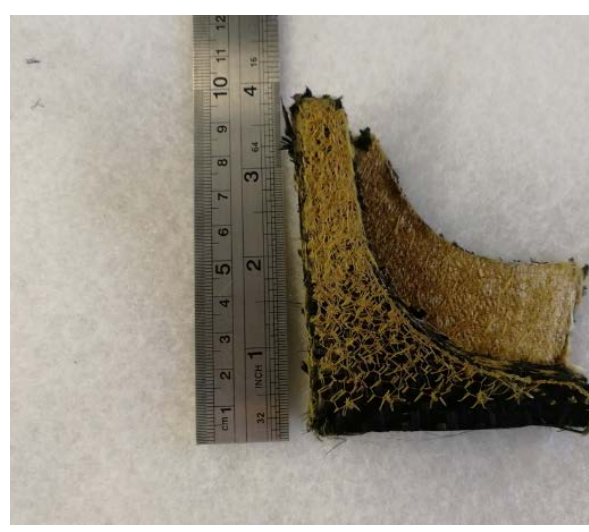

Fig. 3. Work pieces of the blade segment made by the method of directional laying of the reinforcing filler.

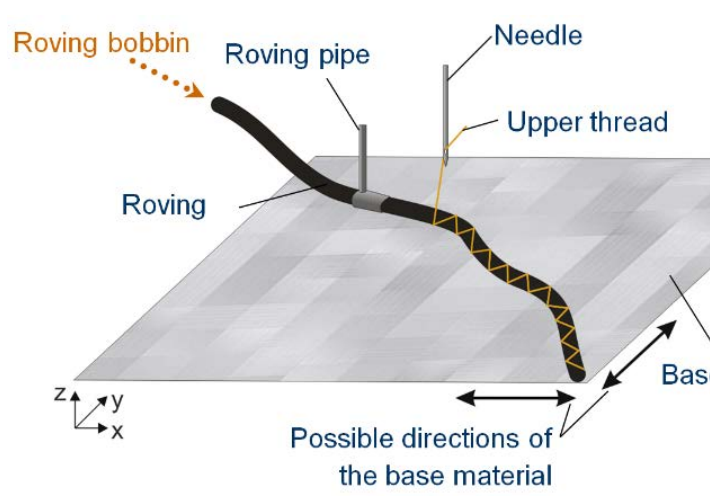

Fig. 4. Principle of laying and fixing the material used in embroidery machines.

It is possible to sew the fibers close to each other with high accuracy and reproducibility, forming a monolayer of the future preform. In each layer, the fibers can be positioned in any given direction and, therefore, this method allows you to fully realize the existing strength properties of the fibers, making a preform with an optimal reinforcement scheme. The auxiliary thread can also be selected from various materials. For products made of composite materials based on carbon fibers, threads made of aramid or thermoplastic polymer are relevant. Each new layer can be sewn on top of the previous one. 
Machines may be able to stack several different types of reinforcing fibers simultaneously. The fiber is sewn to the substrate with a tightening stitch. This fixation of the fibers in the third direction automatically creates a spatial structure of reinforcement. The step and width of the stitch are adjustable, allowing you to create preforms of different surface densities and bulk content of the warp fibers, as well as adjust the content of the stitching fibers. To create relatively safe preform piercing modes, the stitching speed can be adjusted to ensure minimal damage to the main fibers.

Due to the limitation on the thickness embroider the preform which is determined by the strength of the subsidiary strands and the need for further processing of embroidered bags, embroidered is splitting the object into separate packages. For the stripe segment of the impeller of a centrifugal compressor, the most rational division option turned out to be the division into a blade and a disk part. The graphic representation of the embroidery path is shown in figures 5 and 6 . In this case, the blade is divided into 2 symmetrical packages (Fig.7, positions 1,2), and the disk part is divided into 10 packages (Fig.7, positions 3-12).

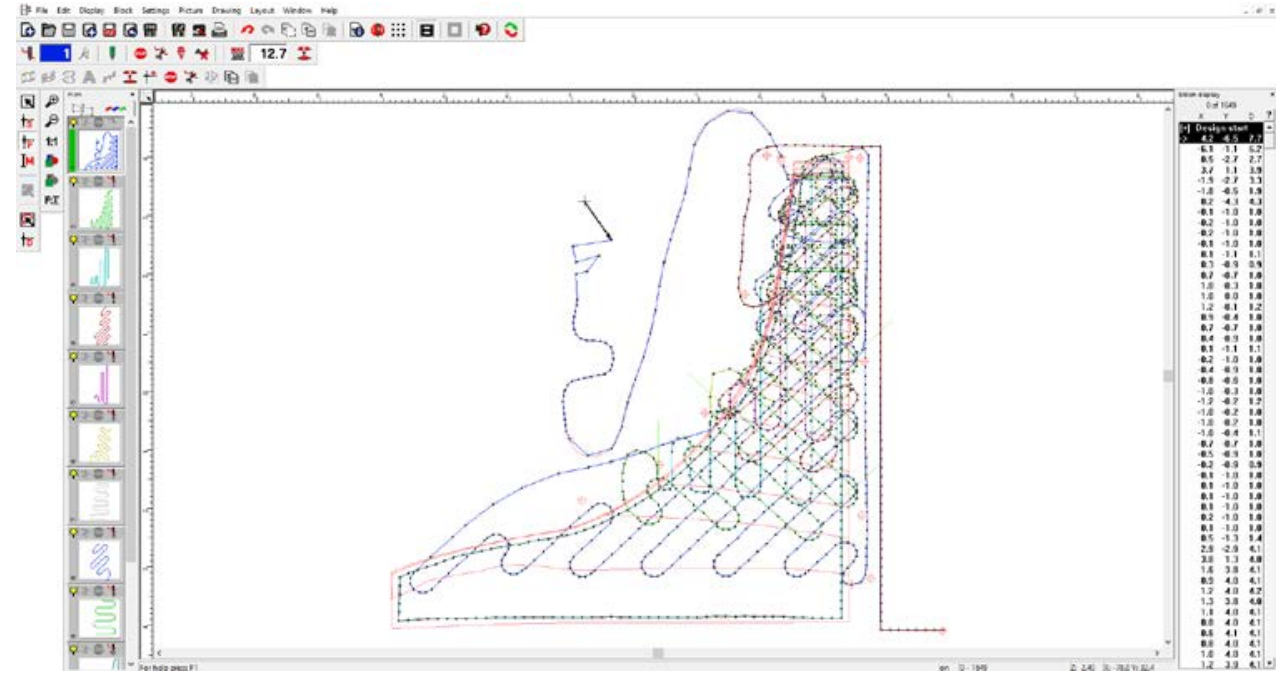

Fig. 5. Graphical representation of the control program for the disk preform stripe.

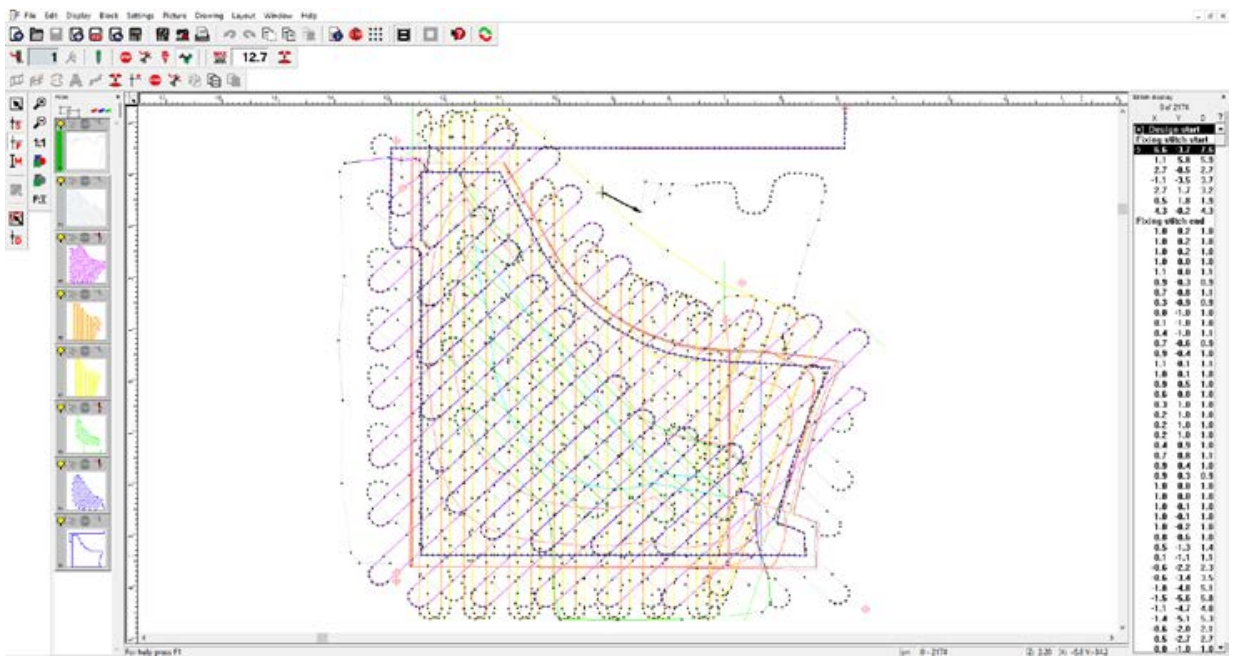

Fig. 6. Graphical representation of the control program for the blade preform patch. 


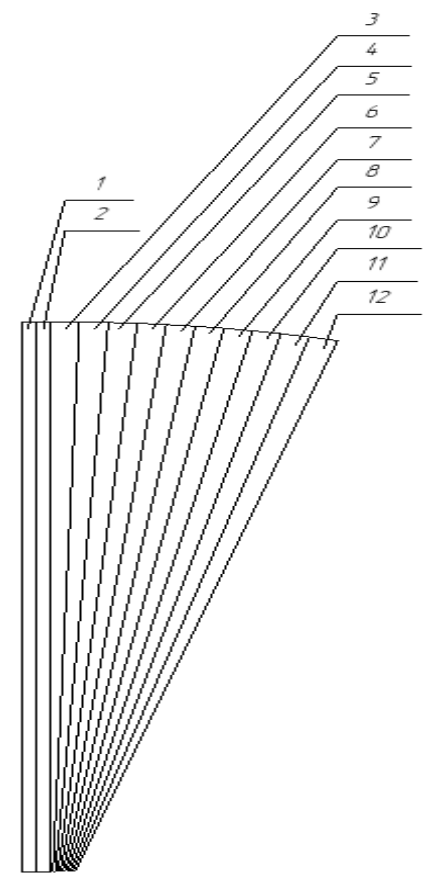

Fig. 7. Diagram of splitting the blade segment into separate sewn packages.

The directions shown in Table 5 were used for manufacturing the blade preforms.

Table 5. Directions for laying the blade preform layers.

\begin{tabular}{|c|c|c|c|c|c|c|c|c|}
\hline № layer's & 1 & 2 & 3 & 4 & 5 & 6 & 7 & 8 \\
\hline Direction, $^{0}$ & 0 & -45 & 45 & 0 & 0 & -45 & 45 & 0 \\
\hline
\end{tabular}

The directions shown in Table 6 were applied to the disk part.

Table 6. Directions for laying layers of the disk part preform.

\begin{tabular}{|c|c|c|c|c|c|c|c|c|c|}
\hline № layer's & 1 & 2 & 3 & 4 & 5 & 6 & 7 & 8 & 9 \\
\hline Direction, $^{0}$ & 45 & -45 & 0 & 45 & 0 & -45 & 90 & 45 & 90 \\
\hline
\end{tabular}

As can be seen from Table.6, the stacking scheme contains layers with a 90-degree orientation. This is due to the need to install power rings and is designed to locally strengthen the back surface of the disk.

The proposed design uses 5 power rings designed to assemble and hold the blade segments in the field of centrifugal forces. Power rings are the most loaded and responsible elements in the design of a compressor made of composite materials. In a ring-shaped part, it is necessary to obtain the theoretical characteristics of a unidirectionally reinforced composite material with the maximum volume content of fiber along the direction of reinforcement and increased rigidity in the radial direction. For the production of power rings, the technology of winding the reinforcing material on a cylindrical mandrel was chosen. The essence of this method is that when winding on a cylindrical mandrel, the necessary pressure to seal the material layers is created by the technological tension of the reinforcing material. When winding a product with a large thickness, there are problems with the distribution of pressure over its cross-section. The fact is that each subsequent layer exerts pressure on the previous one. As a result, the pressure of the layers are summarized. As a result, the closer the material is to the mandrel, the more pressure it 
experiences. The binder, in turn, flows from the high pressure zone to the lower pressure zone, as a result, there is a lack of binder in the center of the part, and an excess at the periphery. This leads to uneven cross-section strength of the part. Therefore, it is necessary to wind the material with variable tension. Contact forming by tension of the harness is convenient to implement and allows you to create a very tight laying of the reinforcing material for cylindrical parts. The high-modulus M46 J Toray carbon fiber is used as a reinforcing material for the power rings.

For assembling and gluing individual segments, special equipment is used that allows you to position the segments relative to each other most accurately. After gluing the segments, the ring grooves are cut under the power rings on the lathe and pasted in. Figure 8 shows the finished impeller of a centrifugal compressor made of polymer composite material.

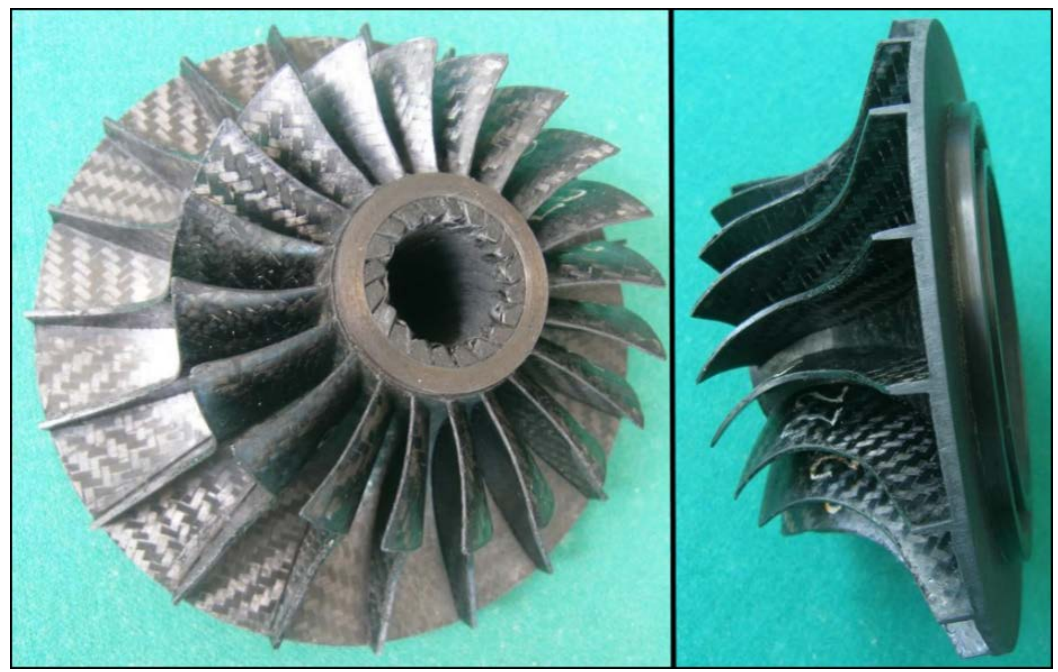

Fig. 8. Impeller of a centrifugal compressor made of polymer composite materials.

\section{Conclusion}

As part of this study, a pilot impeller of a centrifugal compressor was manufactured, which demonstrates the technological possibility of manufacturing a responsible aircraft engine assembly using composite materials for operating temperatures up to $450{ }^{\circ} \mathrm{C}$ based on a heat-resistant thermosetting phthalonitrile binder PN-3M and fillers based on 3D-reinforced carbon fiber preforms UMT-49S for blade segments and high-modulus carbon fiber Toray M-46 J for power rings. The prototype centrifugal compressor impeller was $45 \%$ lighter than its titanium prototype. The use of this technology can reduce the amount of industrial waste by more than 2.5 times.

\section{References}

1. Lanshin A. I., Meleshin V. I., Agarkov N. I., Development of the working layout of a single-stage compressor CDK-3.00.000.00 PZ

2. Vai-Man Lei, Tomoki Kawakubo. A fast method for conjugate heat transfer analysis of centrifugal compressor, (2007) ASME International Mechanical Engineering Congress and Exposition November 11-15, (2007), Seattle, Washington, USA. 
3. Xinqian Zheng, Lei Jin, Tao Du. Effect of temperature on the strength of a centrifugal compressor impeller for a turbocharger, Proc IMechE Part C: J Mechanical Engineering Science

4. German, M. L. Mathematical model for calculating the heat-protection properties of the composite coating "ceramic microspheres-binder" / M. L. German, P. S. Grinchuk // Journal of Engineering Physics and Thermophysics. - (2002). - Vol. 75, N 6. - P. 13011313.

5. K. V. Mikhailovsky, P. V. Prosuntsov, And S. V. Reznik. Development of high-heatconducting polymer composite materials for space structures / / UDC 629.78:620.22

6. E. N. Kablov, G. M. Gunyaev, S. I. Ilchenko. Structural carbon plastics with increased conductivity / / UDC678. 8 